\title{
Gender Differences to the Contextual Factors Questionnaire and Implications for General Practice
}

\author{
Jean Francois Pelletier ${ }^{1,2^{*}}$ \\ ${ }^{1}$ Department of Psychiatry, Yale University School of Medicine, USA \\ ${ }^{2}$ Department of Psychiatry, University of Montreal, Canada
}

"Corresponding author: Jean Francois Pelletier, Assistant Clinical Professor, Department of Psychiatry, Yale University School of Medicine, USA, Tel: 514-251-4000; Email: jeanfrancois.pelletier@yale.edu

Received date: March 17, 2017; Accepted date: March 27, 2017; Published date: April 04, 2017

Copyright: @ 2017 Pelletier JF. This is an open-access article distributed under the terms of the Creative Commons Attribution License, which permits unrestricted use, distribution, and reproduction in any medium, provided the original author and source are credited.

\begin{abstract}
The purpose of this study was to contribute to the revision of the International Classification of Diseases (ICD-10) by exploring how mental health service users and their relatives conceive the influence of the contextual factors, as described in Chap. 21 of the ICD-10, on a) the onset of a mental or behavioral disorder; b) the resurgence of such a disorder; and c) the recovery process. In individual interviews, the 18 main categories of contextual factors proposed by the ICD-11 (beta draft) were discussed through the Contextual Factors Questionnaire (CFQ) by 28 participants who completed the CFQ individually.

This paper presents the results from a t-test that was performed on the data set to assess whether the means for two groups who completed the CFQ were statistically different from each other $(p \leq 0.05)$. On one hand, we found significant differences between users and family members for $A$ ) Housing or the environment $(p=0.01)$, and $B$ ) Personal finances $(p=0.04)$; and for $C)$ Water or nutrition $(p=0.05)$ between male and female participants, on the other hand. In light of the fact that Social or cultural environments were considered to be the most influential of all 18 contextual factors by all aggregated participants, this suggests that male-female differences are related to social norms concerning gender, rather than discrete biological sex categories.
\end{abstract}

Keywords: Gender differences; Health status; Mental health

\section{Background}

Most of the people who would clinically be in need of care regarding mental health problems, do not consult for these reasons [1]. It is frequent, however, that they want to consult about socio-economic or psycho-social conditions that they associate to "problems" that interfere with their daily activities and responsibilities [2]. This project consisted of asking patients with a diagnosis of a mental or behavioral disorder, and their relatives, what is the influence of the contextual factors, if any, on either the onset of such a disorder, its resurgence, or on the recovery process.

These contextual factors influencing health status and contact with health services are gathered in Chap. 21 of the International Classification of Diseases (ICD-10 $0^{\text {th }}$ version). They are provided for occasions when circumstances other than a disease are recorded as "diagnoses" or "problems". As per the World Health Organization [3], this can arise when a person who may or may not be sick encounters the health services for some specific purpose, or to discuss a problem which in itself is not a disease or injury.

This can imply some circumstance or problem which influences the person's health status, circumstance or problem that needs to be borne in mind when the person is receiving care for some illness or injury.

\section{Methods}

The Community-based Participatory Research approach and methods of this project, which consisted in contributing to the revision of the WHO International Classification of Diseases, have been described in this Journal in a previous research paper. The reader can turn to this latter paper for more details on the background and methods of the study which is herein reported.

In brief, the contextual factors influencing health status and contact with health services are gathered in Chap. 21 of the International Classification of Diseases (ICD-10 ${ }^{\text {th }}$ version) $[1,2]$. They are provided for occasions when circumstances other than a disease are recorded as "diagnoses" or "problems".

Twenty-height users of mental health services and relatives of such persons (e.g. parents, friends, siblings) were recruited to take part in individual interviews [4]. Participants talked about the impact of the contextual factors either on a) the onset of a mental or behavioral disorder (Chap. 5 of ICD-10); b) the resurgence of such a disorder; and c) the recovery process. This paper reports the quantitative results for the participants who completed the Contextual Factors Questionnaire (CFQ) in preparation for the interviews. The 18 main categories of contextual factors proposed by the ICD-11 (beta draft) were assessed with this 18 -item CFQ. Participants were invited to tell, on a 6-point Likert scale ( $1=$ no influence at all, $6=$ very influential), how influential these factors were, either for themselves (service users) or according to their relatives who discussed the influence of the contextual factors on their loved one's condition [5]. For comparison among participants (see Pelletier, [2], for the IRB approved recruitment procedure and study design [1]), this 28 participants sample was divided twice in two sub-samples of: $\mathrm{A})$ users $(\mathrm{N}=17)$ and relatives $(\mathrm{N}=11)$; and $\mathrm{B})$ males $(\mathrm{N}=12)$ and females $(\mathrm{N}=16)$. Assuming symmetrical distribution between these independent sub-groups, two-tailed $t$-tests were performed with the Statistical Package for the Social Sciences software 
Citation: Pelletier JF (2017) Gender Differences to the Contextual Factors Questionnaire and Implications for General Practice. J Gen Pract (Los

Page 2 of 5

(SPSS, $24^{\text {th }}$ version), to assess statistical differences among the subsamples $[6,7]$.

\section{Results}

Using a significance level of 0.05 , (Tables 1 and 2) respectively report the results for $\mathrm{A}$ ) the user/relative grouping; and B) the male/ female grouping.

\begin{tabular}{|c|c|c|c|c|c|c|c|c|c|c|c|c|c|}
\hline $\begin{array}{l}\text { Item of the } \\
\text { CFQ }\end{array}$ & $\begin{array}{l}\text { User }(n=17) \text { or } \\
\text { relative }(n=11)\end{array}$ & Mean & SD & $\begin{array}{l}\text { Sig. } \\
\text { tailed }\end{array}$ & Mean & SD & $\begin{array}{l}\text { Sig. } \\
2- \\
\text { tailed }\end{array}$ & Mean & SD & $\begin{array}{l}\text { Sig. 2- } \\
\text { tailed }\end{array}$ & Mean & SD & $\begin{array}{l}\text { Sig. 2- } \\
\text { tailed }\end{array}$ \\
\hline \multirow{2}{*}{$\begin{array}{l}\text { Personal } \\
\text { finances }\end{array}$} & User & 2.6 & 2.62 & \multirow[t]{2}{*}{0.07} & 3.06 & 2.1 & \multirow[t]{2}{*}{0.31} & 4 & 1.84 & \multirow[t]{2}{*}{0.15} & 9.7 & 4.3 & \multirow[t]{2}{*}{$0.04^{*}$} \\
\hline & Relative & 0.9 & 1.7 & & 2.18 & 2.4 & & 2.9 & 2.02 & & 6 & 4.17 & \\
\hline $\begin{array}{l}\text { Water } \quad \text { or } \\
\text { nutrition }\end{array}$ & Relative & 1.2 & 2.14 & & 1.2 & 1.7 & 0.09 & 2.8 & 2.04 & 0.21 & 5.1 & 4.53 & 0.06 \\
\hline \multirow{2}{*}{$\begin{array}{l}\text { Housing or the } \\
\text { environment }\end{array}$} & User & 3.5 & 2.15 & \multirow[t]{2}{*}{$0.00^{* * *}$} & 3.35 & 1.8 & \multirow[t]{2}{*}{$0.01^{* *}$} & 4.1 & 2.09 & \multirow[t]{2}{*}{0.97} & 11 & 4.94 & \multirow[t]{2}{*}{$0.01^{* *}$} \\
\hline & Relative & 0.6 & 1.57 & & 1.36 & 2 & & 4.1 & 2.07 & & 6.1 & 3.96 & \\
\hline \multirow{2}{*}{$\begin{array}{l}\text { Social, } \\
\text { cultural } \\
\text { environments }\end{array}$} & User & 3.8 & 2.35 & \multirow[t]{2}{*}{0.15} & 4.12 & 2 & \multirow[t]{2}{*}{0.11} & 4.7 & 1.27 & \multirow[t]{2}{*}{0.87} & 12.6 & 4.11 & \multirow[t]{2}{*}{0.11} \\
\hline & Relative & 2.6 & 2.02 & & 2.82 & 2.1 & & 4.7 & 1.27 & & 10.1 & 3.56 & \\
\hline \multirow{2}{*}{$\begin{array}{l}\text { Employment/ } \\
\text { unemploymen } \\
\text { t }\end{array}$} & User & 3 & 2.15 & \multirow[t]{2}{*}{$0.02^{*}$} & 3.59 & 2.1 & \multirow[t]{2}{*}{0.17} & 3.9 & 1.87 & \multirow[t]{2}{*}{0.79} & 10.5 & 4.32 & \multirow[t]{2}{*}{0.06} \\
\hline & Relative & 1.1 & 1.76 & & 2.3 & 2.6 & & 4.1 & 2.38 & & 6.9 & 5.22 & \\
\hline \multirow[t]{2}{*}{ Education } & User & 2.1 & 2.41 & \multirow[t]{2}{*}{0.3} & 2.76 & 2.3 & \multirow[t]{2}{*}{0.08} & 3.5 & 1.91 & \multirow[t]{2}{*}{0.97} & 8.3 & 5.41 & \multirow[t]{2}{*}{0.19} \\
\hline & Relative & 1.2 & 1.66 & & 1.18 & 2 & & 3.5 & 2.22 & & 5.6 & 5.03 & \\
\hline \multirow{2}{*}{$\begin{array}{l}\text { Social security } \\
\text { welfare }\end{array}$} & User & 2.4 & 2.55 & 0.07 & 2.59 & 2.3 & 0.35 & 3.7 & 2.32 & 0.48 & 8.7 & 5.02 & 0.33 \\
\hline & Relative & 0.7 & 1.79 & & 1.73 & 2.3 & & 4.3 & 2.1 & & 6.7 & 4.98 & \\
\hline Judicial & User & 0.9 & 1.83 & 0.13 & 1.76 & 2 & 0.12 & 1.6 & 1.94 & 0.39 & 4.2 & 5.23 & 0.12 \\
\hline & Relative & 0 & 0 & & 0.64 & 1.4 & & 0.9 & 2.07 & & 1.6 & 2.25 & \\
\hline Risk factors & User & 3.4 & 2.4 & 0.06 & 3.35 & 2.3 & 0.69 & 3.3 & 2.11 & 0.73 & 10.1 & 6 & 0.44 \\
\hline & Relative & 5 & 1.41 & & 3.73 & 2.5 & & 3 & 2.37 & & 11.7 & 4.71 & \\
\hline Interpersonal & User & 2.8 & 2.25 & 0.39 & 3.35 & 1.9 & 0.58 & 4.2 & 1.78 & 0.4 & 10.3 & 4 & 0.42 \\
\hline & Relative & 3.6 & 2.38 & & 3.8 & 2.2 & & 4.7 & 1.42 & & 11.7 & 5.31 & \\
\hline Someone & User & 3.1 & 2.62 & 0.8 & 3.88 & 2.2 & 0.85 & 3.1 & 2.15 & 0.46 & 10 & 5.06 & 0.68 \\
\hline behavior & Relative & 3.4 & 2.06 & & 3.73 & 2 & & 3.7 & 1.95 & & 10.8 & 5.25 & \\
\hline Someone & User & 1.6 & 2.12 & 0.11 & 2.24 & 2.2 & 0.71 & 2.4 & 2.45 & 0.51 & 6.2 & 6.15 & 0.34 \\
\hline & Relative & 0.5 & 1.04 & & 1.91 & 2.3 & & 1.7 & 2.33 & & 4.1 & 4.23 & \\
\hline Loss or death & User & 2.6 & 2.24 & 0.21 & 2.47 & 2.1 & 0.38 & 3.4 & 2.12 & 0.08 & 8.4 & 5.51 & 0.21 \\
\hline & Relative & 1.4 & 2.46 & & 3.2 & 1.9 & & 1.9 & 1.79 & & 5.9 & 4.23 & \\
\hline Assault & User & 2.6 & 2.62 & 0.62 & 2.76 & 2.6 & 0.52 & 2.9 & 2.47 & 0.4 & 8.2 & 6.03 & 0.75 \\
\hline events & Relative & 3.1 & 2.33 & & 3.4 & 2.1 & & 2 & 2.6 & & 7.6 & 4.76 & \\
\hline Fear & User & 3.5 & 2.7 & 0.49 & 3.29 & 2.5 & 0.73 & 3.1 & 2.62 & 0.58 & 9.9 & 7.31 & 0.73 \\
\hline & Relative & 2.7 & 2.76 & & 3.64 & 2.5 & & 2.6 & 2.58 & & 8.9 & 6.85 & \\
\hline Impairments & User & 2.2 & 2.54 & 0.95 & 1.88 & 2.2 & 0.57 & 2 & 2.37 & 0.45 & 6.1 & 5.56 & 0.85 \\
\hline & Relative & 2.3 & 2.45 & & 2.4 & 2.3 & & 2.7 & 2.16 & & 6.6 & 5.91 & \\
\hline
\end{tabular}


Citation: Pelletier JF (2017) Gender Differences to the Contextual Factors Questionnaire and Implications for General Practice. J Gen Pract (Los Angel) 5: 297. doi:10.4172/2329-9126.1000297

Page 3 of 5

\begin{tabular}{|c|c|c|c|c|c|c|c|c|c|c|c|c|c|}
\hline \multirow[t]{2}{*}{ Interventions } & User & 1.5 & 2.15 & \multirow[t]{2}{*}{0.22} & 2.47 & 2.4 & \multirow[t]{2}{*}{0.52} & 4.9 & 1.48 & \multirow[t]{2}{*}{0.46} & 8.9 & 3.68 & \multirow[t]{2}{*}{0.51} \\
\hline & Relative & 0.6 & 1.12 & & 3.1 & 2.4 & & 4.5 & 1.97 & & 7.9 & 4.51 & \\
\hline \multirow{2}{*}{$\begin{array}{l}\text { Healthcare } \\
\text { system }\end{array}$} & User & 1.8 & 2.33 & \multirow[t]{2}{*}{0.25} & 2.76 & 2.3 & \multirow[t]{2}{*}{0.7} & 4.7 & 1.73 & \multirow[t]{2}{*}{0.89} & 9.2 & 4.4 & \multirow[t]{2}{*}{0.51} \\
\hline & Relative & 0.8 & 1.93 & & 3.1 & 2.1 & & 4.6 & 2.02 & & 8.1 & 4.35 & \\
\hline \multirow[t]{2}{*}{ Total } & User & 45.5 & 26.2 & \multirow[t]{2}{*}{0.11} & 53.9 & 19 & \multirow[t]{2}{*}{0.23} & 63.1 & 18.6 & \multirow[t]{2}{*}{0.47} & 161 & 50.2 & \multirow[t]{2}{*}{0.14} \\
\hline & Relative & 30.9 & 15 & & 44.8 & 19 & & 57.2 & 24.2 & & 131 & 48.1 & \\
\hline
\end{tabular}

Table 1: User-relative differences to the Contextual Factors Questionnaire (CFQ).

\begin{tabular}{|c|c|c|c|c|c|c|c|c|c|c|c|c|c|}
\hline \multirow[b]{2}{*}{$\begin{array}{l}\text { Item of the } \\
\text { CFQ }\end{array}$} & \multirow[b]{2}{*}{$\begin{array}{l}\text { Male }(n=12) \text { or } \\
\text { female }(n=16)\end{array}$} & \multicolumn{3}{|l|}{ Onset } & \multicolumn{3}{|c|}{ Resurgence } & \multicolumn{3}{|c|}{ Recovery } & \multicolumn{3}{|c|}{ All dimensions } \\
\hline & & Mean & SD & $\begin{array}{l}\text { Sig. 2- } \\
\text { tailed }\end{array}$ & Mean & SD & $\begin{array}{l}\text { Sig. 2- } \\
\text { tailed }\end{array}$ & Mean & SD & $\begin{array}{l}\text { Sig. 2- } \\
\text { tailed }\end{array}$ & Mean & SD & $\begin{array}{l}\text { Sig. 2- } \\
\text { tailed }\end{array}$ \\
\hline \multirow{2}{*}{$\begin{array}{l}\text { Personal } \\
\text { finances }\end{array}$} & Male & 2.1 & 2.39 & \multirow{2}{*}{0.78} & 2.42 & 2.1 & \multirow{2}{*}{0.55} & 3.3 & 1.72 & \multirow{2}{*}{0.59} & 7.8 & 4.44 & \multirow{2}{*}{0.71} \\
\hline & Female & 1.8 & 2.51 & & 2.94 & 2.4 & & 3.8 & 2.15 & & 8.5 & 4.74 & \\
\hline \multirow{2}{*}{$\begin{array}{l}\text { Water or } \\
\text { nutrition }\end{array}$} & Male & 1.6 & 2.39 & \multirow{2}{*}{0.8} & 1.42 & 1.4 & \multirow{2}{*}{0.14} & 2.2 & 2.25 & \multirow{2}{*}{$0.00^{*}$} & 5.2 & 4.39 & \multirow{2}{*}{$0.05^{*}$} \\
\hline & Female & 1.8 & 2.29 & & 2.6 & 2.4 & & 4.4 & 1.55 & & 8.7 & 4.48 & \\
\hline \multirow{2}{*}{$\begin{array}{l}\text { Housing or } \\
\text { the } \\
\text { environment }\end{array}$} & Male & 2.5 & 2.2 & \multirow{2}{*}{0.84} & 2.42 & 1.8 & \multirow{2}{*}{0.74} & 3.8 & 2.04 & \multirow{2}{*}{0.55} & 8.8 & 4.79 & \multirow{2}{*}{0.78} \\
\hline & Female & 2.3 & 2.6 & & 2.69 & 2.4 & & 4.3 & 2.09 & & 9.3 & 5.5 & \\
\hline \multirow{2}{*}{$\begin{array}{l}\text { Social, } \\
\text { cultural } \\
\text { environments }\end{array}$} & Male & 3.5 & 1.78 & \multirow{2}{*}{0.73} & 3.67 & 1.6 & \multirow{2}{*}{0.9} & 4.3 & 1.37 & \multirow{2}{*}{0.21} & 11.5 & 3.34 & \multirow{2}{*}{0.91} \\
\hline & Female & 3.2 & 2.64 & & 3.56 & 2.5 & & 4.9 & 1.12 & & 11.7 & 4.59 & \\
\hline \multirow{2}{*}{$\begin{array}{l}\text { Employment/ } \\
\text { unemploymen } \\
t\end{array}$} & Male & 2.5 & 2.15 & \multirow{2}{*}{0.61} & 3.17 & 2.2 & & 3.6 & 2.31 & & 9.3 & 4.48 & \\
\hline & Female & 2.1 & 2.27 & & 3.07 & 2.5 & & 4.3 & 1.79 & & 8.9 & 5.38 & \\
\hline & Male & 1 & 1.35 & & 1.17 & 1.9 & & 2.8 & 2.17 & & 5 & 4.59 & \\
\hline & Female & 2.3 & 2.52 & & 2.88 & 2.4 & & 4 & 1.73 & & 8.9 & 5.39 & \\
\hline Social & Male & 1.3 & 2.26 & & 1.42 & 2 & & 3.8 & 2.22 & & 6.4 & 3.2 & \\
\hline welfare & Female & 2.1 & 2.5 & & 2.88 & 2.4 & & 4 & 2.28 & & 9 & 5.88 & \\
\hline Judicial & Male & 0.3 & 0.62 & & 0.83 & 1.5 & & 1.1 & 2.07 & & 2.2 & 2.95 & \\
\hline system & Female & 0.8 & 1.88 & & 1.69 & 2.1 & & 1.5 & 1.97 & & 3.9 & 5.29 & \\
\hline Did fata & Male & 3.9 & 2.02 & 000 & 3.33 & 2.2 & 075 & 3.1 & 1.98 & 005 & 10.3 & 4.33 & 078 \\
\hline 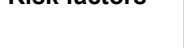 & Female & 4.1 & 2.36 & 0.01 & 3.63 & 2.5 & 0.15 & 3.3 & 2.38 & 0.00 & 11 & 6.37 & 0.10 \\
\hline Interpersonal & Male & 3.8 & 2.04 & 012 & 3.75 & 1.8 & & 3.9 & 1.38 & & 11.5 & 4.1 & \\
\hline interactions & Female & 2.5 & 2.37 & 0.10 & 3.33 & 2.2 & & 4.8 & 1.77 & & 10.4 & 4.88 & 0.00 \\
\hline Someone & Male & 3.4 & 1.83 & & 3.25 & 1.9 & & 2.8 & 2.08 & & 9.5 & 5.02 & 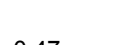 \\
\hline behavior & Female & 3.1 & 2.77 & & 4.25 & 2.2 & & 3.8 & 2.02 & & 10.9 & 5.16 & \\
\hline Someone & Male & 0.9 & 1.44 & 058 & 2.58 & 2.2 & 034 & 1.9 & 2.15 & 072 & 5.4 & 4.31 & 0.96 \\
\hline else's IIlness & Female & 1.3 & 2.12 & & 1.75 & 2.3 & & 2.3 & 2.6 & & 5.3 & 6.36 & \\
\hline Loss or death & Male & 1.7 & 2.15 & 35 & 2.58 & 1.9 & 073 & 2.4 & 1.62 & 039 & 6.7 & 3.58 & 051 \\
\hline of a close one & Female & 2.5 & 2.5 & & 2.87 & 2.2 & & 3.1 & 2.42 & & 8 & 6.08 & \\
\hline
\end{tabular}




\begin{tabular}{|c|c|c|c|c|c|c|c|c|c|c|c|c|c|}
\hline \multirow{2}{*}{$\begin{array}{l}\text { Assault or } \\
\text { harmful } \\
\text { events }\end{array}$} & Male & 2.8 & 2.45 & \multirow{2}{*}{0.96} & 2.75 & 2.3 & \multirow{2}{*}{0.64} & 2 & 2.49 & \multirow{2}{*}{0.29} & 7.5 & 3.63 & \multirow{2}{*}{0.71} \\
\hline & female & 2.8 & 2.6 & & 3.2 & 2.5 & & 3.1 & 2.5 & & 8.3 & 6.64 & \\
\hline \multirow{2}{*}{ Fear } & Male & 2.9 & 2.71 & \multirow{2}{*}{0.67} & 3.17 & 2.5 & \multirow{2}{*}{0.64} & 1.8 & 2.25 & \multirow{2}{*}{0.06} & 7.9 & 6.56 & \multirow{2}{*}{0.31} \\
\hline & Female & 3.4 & 2.75 & & 3.63 & 2.6 & & 3.7 & 2.58 & & 10.7 & 7.32 & \\
\hline \multirow{2}{*}{$\begin{array}{l}\text { Impairments } \\
\text { or restrictions }\end{array}$} & Male & 2.4 & 2.31 & \multirow{2}{*}{0.77} & 2.25 & 2.3 & \multirow{2}{*}{0.72} & 1.9 & 2.39 & \multirow{2}{*}{0.5} & 6.4 & 4.66 & \multirow{2}{*}{0.92} \\
\hline & Female & 2.1 & 2.64 & & 1.93 & 2.2 & & 2.5 & 2.23 & & 6.2 & 6.3 & \\
\hline \multirow{2}{*}{ Interventions } & Male & 0.6 & 1.08 & \multirow{2}{*}{0.14} & 1.83 & 2.1 & \multirow{2}{*}{0.09} & 4.6 & 1.93 & \multirow{2}{*}{0.66} & 7 & 3.41 & \multirow{2}{*}{0.08} \\
\hline & Female & 1.6 & 2.19 & & 3.4 & 2.4 & & 4.9 & 1.5 & & 9.7 & 4.08 & \\
\hline \multirow{2}{*}{$\begin{array}{l}\text { Healthcare } \\
\text { system }\end{array}$} & Male & 0.5 & 1 & \multirow{2}{*}{$0.04^{*}$} & 2.08 & 1.6 & \multirow{2}{*}{0.08} & 4.5 & 1.93 & \multirow{2}{*}{0.79} & 7.1 & 2.61 & \multirow{2}{*}{0.07} \\
\hline & Female & 2.2 & 2.62 & & 3.53 & 2.4 & & 4.7 & 1.78 & & 10.1 & 4.97 & \\
\hline \multirow{2}{*}{ Total } & Male & 37.6 & 18.12 & \multirow{2}{*}{0.68} & 45.08 & 16.7 & \multirow{2}{*}{0.22} & 53.9 & 23.15 & \multirow{2}{*}{0.13} & 135.4 & 40.12 & \multirow{2}{*}{0.22} \\
\hline & Female & 41.4 & 27.02 & & 54.31 & 21 & & 65.9 & 17.74 & & 159.5 & 56.3 & \\
\hline
\end{tabular}

Table 2: Male-female differences to the Contextual Factors Questionnaire (CFQ).

As per Table 1, the most significant difference for all dimensions combined $(p=0.01)$ is between users and family members, and related to Housing or the environment ( $\mathrm{p}=0.00$ for onset, and $\mathrm{p}=0.01$ for resurgence). Service users see this contextual factor to be more influential than family members do. This is also the case with Employment/unemployment ( $\mathrm{p}=0.02$ for onset), and for Personal finances ( $\mathrm{p}=0.04$ for all dimensions combined). In terms of malefemale differences (Table 2), the most significant difference is for Water or nutrition ( $\mathrm{p}=0.00$ and $\mathrm{p}=0.05$, respectively for recovery, and for all dimensions combined) [8]. The male-female difference is also significant in terms of Education $(\mathrm{p}=0.05)$, whereas this difference is $\mathrm{p}=0.04$ with respect to the Healthcare system $[8,9]$. It is with females that these two latter contextual factors are perceived to be more influential, respectively with regards to resurgence, and onset. Female participants also perceive Water or nutrition to be more influential than males do in terms of recovery and when all onset, resurgence, and recovery dimensions remain combined.

Thus, among all 18 contextual factors, as mentioned above, we found statistically significant differences between users and family members for A) Housing or the environment and B) Personal finances; and for $\mathrm{C}$ ) Water or nutrition, this time in terms of male-female differences [10]. At the other end of the spectrum, the least statistically significant differences are for $\mathrm{D})$. Someone else's illness $(\mathrm{p}=0.96), \mathrm{E})$ Impairments or restrictions $(\mathrm{p}=0.92)$, and $\mathrm{F}$ ) Social, cultural environments ( $\mathrm{p}=0.91$ ), each time in terms of male-female differences.

\section{Discussion}

Indeed, as previously reported [1], the Social or cultural environments factor is considered to be the most influential contextual factor for all onset, resurgence, and recovery dimensions combined. There seems also be a consensus that Impairments, limitations or restrictions ( $\mathrm{p}=0.85$ for user-relative; $\mathrm{p}=0.92$ for male-female) and someone else's illness ( $p=0.68$ for user/relative; $p=0.96$ for male/ female) are the least influential, with the exception of the Judicial system.
This is the least influential for each dimension separately and therefore, for all dimensions combined, but with much sharper discrepancies between users and relatives $(p=0.12)$, as well as between males and females $(\mathrm{p}=0.31)$.

Users consider their socio-economic status to be more influential than relatives do (e.g. finances, housing, and employment).

And in light of the fact that Social or cultural environments were considered to be the most influential of all 18 contextual factors by all aggregated participants, this suggests that male-female differences are related to social norms concerning gender, rather than discrete biological sex categories. In effect, sex typically refers to a set of biological attributes, whereas gender refers to socially constructed social roles [3]. Since gender influences how people perceive themselves, it also influences help-seeking intentions [4].

For instance, self-stigma is related to cultural and gender-role norms, the masculinity ideology and help-seeking intentions being known to be mediated by attitude and subjective norm [5], with the consequence that men are less likely than women to seek help for their mental health [6,7]. Moreover, symptoms of depression for men are often expressed through somatic symptoms like fatigue or sleep problems [8-15], for which people commonly show up in general practice.

\section{Conclusion}

These results highlight the relevance for primary care providers to assess the contextual factors that may be at play in the recovery process of their patients also with mental health needs. Further research will be needed to test the reliability and psychometric validity of the Contextual Factors Questionnaire (CFQ) when the WHO will have released the final ICD- $11^{\text {th }}$ version.

The actual CFQ beta-draft nevertheless proved to be useful. Results to such a survey, and doctor-patient discussion of these results, can be used in general practice to identify specific support needs and to provide gender-sensitive encouragement to diverse patients, for them to seek help and support, once the need for it is recognized. 
Citation: Pelletier JF (2017) Gender Differences to the Contextual Factors Questionnaire and Implications for General Practice. J Gen Pract (Los Angel) 5: 297. doi:10.4172/2329-9126.1000297

Page 5 of 5

\section{Acknowledgement}

This study was supported by grants from the Douglas Mental Health University Institute, the Montreal Mental Health University Institute, and the Quebec Mental Health University Institute, province of Quebec, Canada. This multi-site study was approved by the Institutional Review Board of the Montreal Mental Health University Institute.

\section{References}

1. Wallerstein NB, Duran B (2006) Using community-based participatory research to address health disparities. Health Promot Pract 7: 312-323.

2. Pelletier JF (2017) Contribution of service users to the revision of the WHO International Classification of Diseases: should recovery also apply to General Practice? J Gen Pract (Los Angel) 5: 289.

3. World Health Organization (1999) International Statistical Classification of Diseases and Related Health Problems (10th Revision). Geneva.

4. (2017) Canadian Institutes of Health Research Sex, Gender and Health Research Guide: A Tool for CIHR Applicants.

5. Tedstone Doherty D, Kartalova-O'Doherty Y (2010) Gender and selfreported mental health problems: predictors of help-seeking from a general practitioner. Br J Health Psychol 15: 213-228.

6. Bayer J, Peay M (1997) Predicting intentions to seek help from professional mental health services. Aust N Z J Psychiatry 31: 504-513.
7. Sirey J, Bruce L, Alexopoulos G, Perlick D, Raue P, et al. (2001) Perceived stigma as a predicator of treatment discontinuation in younger and older outpatients with depression. Am J Psychiatry 158: 479-481.

8. Mojtabai R (2013) Americans' attitudes toward mental health treatment seeking: 1990-2003. Psychiatr Serv 58: 642-651.

9. Smith JP, Tran GQ, Thompson RD (2008) Can the theory of planned behavior help explain men's psychological help-seeking? Evidence for a mediation effect and clinical implications. Psychol Men Masculin 9: 179-192.

10. Rabinowitz J, Gross R, Feldman D (1999) Correlates of a perceived need for mental health assistance and differences between those who do and do not seek help. Soc Psychiatry Psychiatr Epidemiol 34: 141-146.

11. Wilkins D, Kemple M (2011) Delivering male: effective practice in male mental health. London: Mind.

12. Montano CB (1994) Recognition and treatment of depression in a primary care setting. J Clin Psychiatry 55: 18 .

13. Alderson SL, Foy R, Glidewell L, House AO (2014) Patients understanding of depression associated with chronic physical illness: a qualitative study. BMC Fam Pract 15: 37.

14. Cheshire A, Peters D, Ridge D (2016) How do we improve men's mental health via primary care? An evaluation of the Atlas Men's Well-being Pilot Programme for stressed/distressed men. BMC Fam Pract 17:13.

15. Rogler L, Cortes D (1993) Help-seeking Pathways: A Unifying Concept in Mental Health Care. Am J Psychiatry 150: 554-561. 